\title{
Development of an integral assessment approach of health status in patients with obstructive airway diseases: the CORONA study
}

\author{
This article was published in the following Dove Press journal: \\ International Journal of COPD \\ 6 November 2015 \\ Number of times this article has been viewed
}

\author{
Edmée FMM van den Akker \\ Alex J van ' $t \mathrm{Hul}^{2}$ \\ Niels H Chavannes ${ }^{3}$ \\ Gert-Jan Braunstahl' \\ Alie van Bruggen' \\ Maureen PMH Rutten-van \\ Mölken ${ }^{4}$ \\ Johannes CCM in 't Veen' \\ 'Department of Pulmonary Diseases, \\ STZ Center of Excellence for \\ Asthma \& COPD, Sint Franciscus \\ Gasthuis, Rotterdam, ${ }^{2}$ Department \\ of Pulmonary Disease, Radboud \\ University Medical Center, Nijmegen, \\ ${ }^{3}$ Department of Public Health and \\ Primary Care, Leiden University \\ Medical Center, Leiden, ${ }^{4}$ Department \\ of Health Care Policy and \\ Management, Institute of Medical \\ Technology Assessment, Erasmus \\ University Rotterdam, Rotterdam, \\ the Netherlands
}

Correspondence: Edmée FMM van den Akker

Department of Pulmonology, STZ Center of Excellence for Asthma \& COPD, Sint Franciscus Gasthuis, PO Box 10900 , 3004 BA Rotterdam, the Netherlands

$\mathrm{Tel}+3$ I 6 II 536439

Email e.vandenakker@sfg.nl
Background: Traditional assessment of patients with obstructive lung diseases (asthma and chronic obstructive pulmonary disease; COPD) relies on physiological tests. The COPD and Asthma Rotterdam Integrated Care Approach (CORONA) study aims to develop a diagnostic pathway with a more comprehensive approach to the assessment of patients with asthma and COPD in secondary care.

Methods: An eight-step method was used to develop and implement the pathway for patients with asthma or COPD referred to an outpatient hospital setting.

Results: The diagnostic pathway consists of an evidence-based set of measurements prioritized by a Delphi procedure. The pathway incorporates three innovative diagnostics: the metronomepaced hyperventilation test to measure dynamic hyperinflation, an activity monitor to objectively evaluate physical activity in daily life, and the Nijmegen Clinical Screening Instrument as a comprehensive assessment tool to acquire detailed insight into symptoms, functional limitations, and quality of life.

Conclusion: An innovative diagnostic pathway was developed and implemented for patients with obstructive lung diseases referred to secondary care. As this pathway aims to provide a comprehensive analysis of health status, it focuses on biomedical aspects and also reviews behavioral aspects that further elucidate the patient's health status. The added value of the diagnostic pathway needs to be determined from both an organizational perspective and from the individual patient's viewpoint.

Keywords: diagnostic pathway, integrated care, comprehensive assessment, shared decisionmaking, health status

\section{Introduction}

Chronic obstructive pulmonary disease (COPD) is associated with a complex and heterogenic symptomatology, which includes pulmonary signs as dyspnea and cough, as well as general aspects such as fatigue, exercise limitation, and mood disturbances. However, airway obstruction is only partially responsible for these symptoms and only moderate correlations are found between airway obstruction and symptom severity. Partly independent of airway obstruction, other pulmonary function impairments like dynamic hyperinflation and disturbances in gas exchange might be present, adding to the symptom load. ${ }^{1}$ Moreover, extrapulmonary manifestations, such as peripheral muscle dysfunction and reduced physical activity, are important consequences of COPD and may add to symptom severity, exercise limitation, and reduced quality of life. ${ }^{2}$ Finally, illness perceptions and cognitions, self-management abilities, and adaptation to the 
challenges of a chronic condition appear to be important moderators of the burden of disease in COPD. ${ }^{3}$

A similar line of reasoning applies to patients with bronchial asthma: both patients and physicians tend to underestimate severity, while multiple factors such as allergies, bronchial hyperactivity, adherence to medication, overweight, a low level of physical activity, functional deficits, and mood moderate the impact on the burden of disease. ${ }^{4}$

These different aspects of the burden of disease are recognized in the concept "health status". Theoretical models and empirical studies defined health status as an overall concept, covering physiological functioning, symptoms, functional limitations, and quality of life as main domains. ${ }^{5}$ These main domains are further subdivided into many more concrete conceptually distinct subdomains. ${ }^{6}$ In patients with COPD, a detailed overview of their health status by means of an integral assessment approach is essential for patienttailored treatment. ${ }^{6,7}$ This conceptualization of health status is very much in line with the concept "burden of disease" as it is defined in the Dutch Standards of Care for patients with asthma and COPD. ${ }^{8}$ This concept stresses the importance of the patient's own experiences of the disease. ${ }^{9}$

In response to the complexity and heterogeneity of the impaired health status, it has been proposed to use a comprehensive, holistic approach to patients with obstructive lung $\operatorname{disease}^{9,10}$ and adopt a personal-goal-oriented patient care model. ${ }^{11}$ Although the international guidelines for asthma and COPD describe which aspects might be included in an integral assessment of health status in patients with asthma and COPD, they lack details on how to operationalize and deploy them. ${ }^{1,12}$ Some suggestions are provided for monitoring parameters, but are limited to the physical aspects of disease and do not address psychosocial and behavioral aspects. $^{9,11}$

Therefore, the present study aims to design a structured diagnostic pathway for patients with asthma and COPD referred to secondary care. The objective is to make a comprehensive integral assessment of the health status and to identify behavioral aspects contributing to the impaired health status. ${ }^{8,13}$ This will guide the formulation of a personalized treatment plan for each patient, including pharmacological and nonpharmacological interventions. Moreover, the care pathway should be complementary and additional to the care provided in primary care.

\section{Development of a diagnostic pathway}

We used an eight-step method to develop, implement, evaluate, and continuously monitor the diagnostic pathway for patients with asthma and COPD. ${ }^{14}$ This method describes a multidisciplinary approach, which aims to translate evidencebased knowledge into clinical practice and was successfully applied in designing an evidence-based pathway for patients with a COPD exacerbation. ${ }^{14}$ Figure 1 presents the eight steps

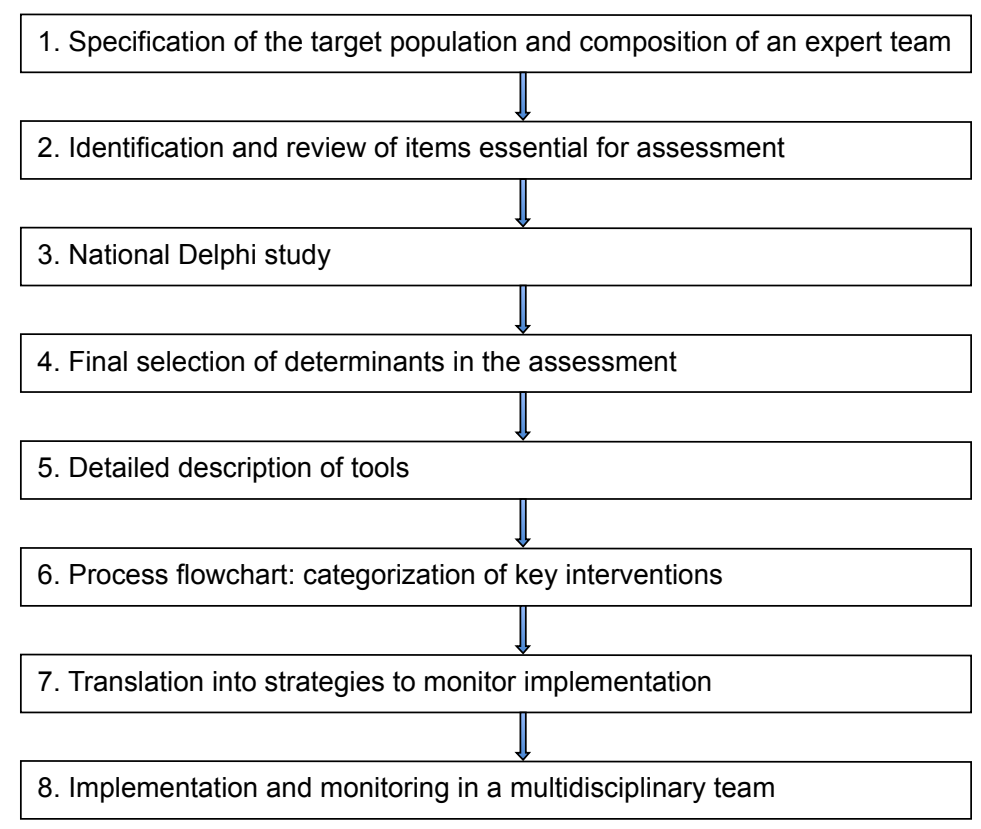

Figure I Eight-step method used for development of the outpatient-based diagnostic pathway for patients with asthma and chronic obstructive pulmonary disease. 
adapted for our diagnostic pathway; each step is described separately in the following section.

\section{Step I: specification of the target population and composition of an expert team}

The care population was specified as "all patients with asthma and COPD referred to the pulmonologist, either by their general practitioner or another medical specialist". We choose a combined diagnostic pathway for both asthma and COPD because after the age of 40, distinguishing between asthma with chronic airflow limitation and COPD becomes problematic and many patients have features of both asthma and COPD. ${ }^{1,12}$ In addition, the process of unraveling behavioral aspects that contributes to the impaired health status (an important aspect of our diagnostic pathway) is diagnosis independent and relevant for all patients with chronic disease. Another aspect is the economies of scale, ie, costs per patient reduce when using facilities for a larger target group. ${ }^{15}$

Delegates of health care professionals and of hospital management were included in the expert team to guarantee a broad support during the development and implementation process. This multidisciplinary team included a pulmonologist, physician assistant, specialist nurse, physical therapist, lung function technician, members of the respiratory management unit, and a member of the administrative staff of the pulmonology department. The group was divided into two smaller teams: one responsible for the content of the diagnostic pathway and the second responsible for logistics.

An external group of experts from primary care (located within the referral area of the hospital) was assembled to address the embedding of this diagnostic assessment in primary care before referral and after discharge of the patient. Criteria for referral and discharge were defined in conjunction with the external group according to national guidelines. A template for referral to the diagnostic pathway was developed.

\section{Step 2: identification and review of items essential for assessment}

The starting point for the diagnostic pathway was the Dutch Standard of Care for patients with asthma and COPD. These standards are based on the international literature, which apply a model to understand the health status of a patient based on four domains: physiological impairment, symptoms, functional impairment, and quality of life (including health-related quality of life)..$^{5,8,16}$ For each of these domains, we reviewed the available diagnostic tests, questionnaires, and severity indicators. ${ }^{17,18}$ We also reviewed which measurements were recommended in international and national guidelines for primary and secondary care. ${ }^{1,8,12,16}$ Furthermore, input was incorporated from the pulmonary rehabilitation literature in which a comprehensive assessment of the patient forms the basis of the management and selection of treatment modules. ${ }^{19}$

\section{Step 3: national Delphi study}

Although pulmonologists generally acknowledge the importance of the aforementioned domains, there is no consensus on which specific components should be part of an integral assessment in the diagnostics process in an outpatient pulmonary department. Therefore, we performed a two-round Delphi process to identify the collective opinion. ${ }^{20}$ A nationwide panel of 153 stakeholders, recommended by their respective professional associations, was formed. The panel included pulmonologists, respiratory nurses, general practitioners, advisors of health insurance companies, and delegates of patient organizations. Agreement between responders on the clinical importance of the various components of the diagnostic assessment was found in 29 of the 41 components (Table S1). Outcomes of the first round were used to develop the diagnostic pathway. The final results of the total Delphi process will be published more extensively elsewhere.

\section{Step 4: final selection of components}

The next step for the expert team was to develop a flowchart of activities required to assess the components that cover all four domains consistent with the Delphi round. The long list derived in Step 3 formed the basis for the final selection by the expert team. A justification for their selection is that this set should 1) reflect disease severity, 2) estimate the risk of future events, and 3) describe the impact of the disease on health status.

\section{Step 5: detailed description of tools}

The expert team selected specific tools to assess the aforementioned components (subdomains) of the four main domains; these are systematically screened in every patient in the diagnostic trajectory. The tools that measure the components in the integral assessment approach can be divided into diagnostic measurements that are discriminatory of specific pathologies or disease phenotypes, or tools that measure the impact of the disease.

Table 1 presents the selected tools to measure the four main domains and their subdomains. 
Table I Selected instruments and subdomains measured for each of the four domains of health status

\section{COPD}

I) Main domain physiological functioning

- History: eg, smoking habit, exacerbations, comorbidity

- BMI

- Capillary blood gases

- Spirometry and flow volume curve, reversibility

- Metronome-paced hyperventilation (MPH) test

- Chest X-ray (age $>40$ years)

2) Main domain symptoms

- Medical Research Council (MRC) dyspnea scale

- CCQ: subdomain symptoms

- NCSI: subdomain dyspnea and fatigue

3) Main domain functional limitations

- CCQ: subdomain functional state

- Dynaport triaxial accelerometer: actual activity level

- NCSI: subdomains subjective and behavioral impairment

4) Main domain quality of life

- CCQ: subdomain emotions

- NCSI: subdomains general quality of life, health-related quality of life, and satisfaction with relations

\section{Asthma}

I) Main domain physiological functioning

- History: eg, smoking habit, exacerbations, comorbidity

- BMI

- Capillary blood gases

- Spirometry and flow volume curve, reversibility

- Metronome-paced hyperventilation (MPH) test

- Chest X-ray

- Allergic assessment (RAST and blood eosinophils and total lgE)

- Nitric oxide (FeNO)

2) Main domain symptoms

- ACQ

- Medical Research Council (MRC) dyspnoea scale

- NCSI: subdomains dyspnea and fatigue

3) Main domain functional limitations

- ACQ

- Dynaport triaxial accelerometer: actual activity level

- NCSI: subdomains subjective and behavioral impairment

4) Main domain quality of life

- NCSI: subdomains general quality of life, health-related quality of life, and satisfaction with relations

Abbreviations: RAST, radioallegrosorbent test; IgE, Immunoglobulin E; CCQ, Clinical COPD Questionnaire; NCSI, Nijmegen Clinical Screening Instrument; BMI, body mass index; ACQ, Asthma Control Questionnaire.

\section{Physiological components in COPD and asthma}

The most commonly encountered and modifiable risk factor that determines physiological dysfunction with accelerated decline of lung function is tobacco smoking. ${ }^{1,12}$ Exacerbations and comorbidities are registered in a structured way as both are important determinants of health status, as well as the prognosis of asthma and COPD. ${ }^{1,12,21}$ There is an association between a low body mass index (BMI) and poor prognosis in COPD and an association between a high BMI and poorer asthma control..$^{22,23}$
Capillary blood gas values are obtained to evaluate pulmonary gas exchange. Although $\mathrm{PaO}_{2}$ may be underestimated by this technique, levels of $\mathrm{PaCO}_{2}$ and oxygen saturation can be reliably measured. ${ }^{24}$

Spirometry and a flow-volume curve are obtained, using internationally accepted methods and references, to evaluate the presence and severity of airway obstruction and reversibility. The forced expiratory volume in 1 second $\left(\mathrm{FEV}_{1}\right)$ and its bronchodilator response indicates airflow obstruction and hyperresponsiveness. In COPD, severity of airflow obstruction is an independent risk factor for impaired health status and mortality. ${ }^{25}$

To evaluate the presence, severity, and reversibility of dynamic hyperinflation, the metronome-paced hyperventilation (MPH) test is applied. Dynamic hyperinflation is an important measure of pulmonary impairment that affects symptoms and is easily measured with the MPH test. ${ }^{26}$ Changes in lung volumes can occur without changes in $\mathrm{FEV}_{1}$ and are more closely related to exercise performance than $\mathrm{FEV}_{1}{ }^{26}{ }^{26}$ Furthermore, hyperinflation is an important target for bronchodilator therapy.

In all patients aged $>40$ years, a chest X-ray is obtained to enable evaluation of relevant respiratory comorbidity and exclude alternative diagnoses. ${ }^{1,12}$

A radioallegrosorbent test (RAST) is performed in patients aged $<40$ years or patients suspected of having asthma. The RAST is a radioimmunoassay test to detect specific IgE antibodies to known or suspected allergens to guide an allergic predisposition. ${ }^{12}$

Nitric oxide levels are measured in patients suspected of having asthma or aged $<40$ years to assess the level of inflammation. This is a noninvasive, simple, and safe method to determine airway inflammation. A level of $>50 \mathrm{ppb}$ confirms the diagnosis of asthma. ${ }^{27}$

\section{Symptoms associated with COPD and asthma}

The most frequently reported symptom in COPD is breathlessness. The Medical Research Council (MRC) dyspnea questionnaire is a simple and standardized scale that measures functional disability due to breathlessness in patients with COPD. ${ }^{28}$

The Clinical COPD Questionnaire (CCQ) is developed to measure the health status in patients with COPD and consists of three separate domains: symptoms, functional state, and mental state. The Asthma Control Questionnaire (ACQ) measures the level of asthma control. These tools can be used to estimate disease severity, assess functional performance, and response to therapy. ${ }^{29,30}$ 
To provide a valid, reliable, and more detailed description of general and disease-specific symptoms in patients with COPD and asthma, we selected the Nijmegen Clinical Screening Instrument (NCSI). ${ }^{3}$ The NCSI is an empirically composed battery of existing instruments providing a quick but highly detailed assessment of the main domains: symptoms (subdomains; dyspnea, fatigue, and dyspnea-related emotions), the main domain functional impairment (subdomains: subjective and behavioral impairment), and the main domain quality of life (subdomains: general quality of life, health-related quality of life, and satisfaction with relationships). For each subdomain normative data indicate "normal functioning", "mild problems", or "severe problems". ${ }^{3}$ Thus the NCSI also provides information about a patient's disease perception and shows the discrepancy between the severity of the physiological disorder on one hand and the severity of symptoms, functional impairment, and quality of life on the other. Results are presented graphically and an incorporated intervention (ie, discussing the graphical presentation of results in a semistructured way) helps formulate patienttailored treatment goals and increase the patient's motivation to adopt adequate health behaviors and, thereby, activates adequate self-management. ${ }^{3}$

\section{Functional limitations in COPD and asthma}

The functional performance in COPD can easily be assessed with the functional status domain of the CCQ and with the subjective and behavioral impairment subdomains of the NCSI. ${ }^{6,29}$ To objectively measure physical activity over 1 week, the Dynaport triaxial accelerometer was selected; this activity monitor assesses standardized and common physical activities in the range of intensity relevant to patients. Changes in walking speed and daily lying time are measured with a high level of accuracy. ${ }^{31}$

\section{Quality of life in COPD and asthma}

To measure quality of life in patients with COPD, the CCQ was selected. ${ }^{32,33}$ The NCSI has added value as it also measures general quality of life, health-related quality of life, and satisfaction with relations. ${ }^{30}$

\section{Step 6: process flowchart: categorization of key measurements}

Finally, the selected measurements were positioned in a diagnostic pathway that had to meet the following four requirements:

1) It had to comply with the principles of lean management; the pathway should enable a comprehensive analysis of the health status with the least possible diagnostic tests. It should optimize and not maximize integral assessment;

2) As disease severity in asthma and COPD changes over time, the pathway should facilitate "shared care" between primary and secondary care. Therefore, algorithms were developed to refer patients back to primary care if treatment goals were reached, or to keep them in secondary care until predefined thresholds (as defined in the Dutch standard of Care) were reached; $;, 16$

3) The diagnostic pathway aimed at optimizing integral assessment to improve self-management, patient satisfaction, and adherence to treatment and health outcomes. This requires the active involvement of patients, for which we implemented personal goal setting using shared decision-making;

4) Finally, the set of measurements had to be feasible in a clinical setting for patients and for the hospital organization.

The basic diagnostic trajectory gives an in-depth view of the health status. However, in some patients additional assessment may be necessary, eg, additional pulmonary function testing, pulmonary imaging, or referral to another medical discipline. In addition, in patients with a low physical activity measured over 1 week, defined as steps/day $<5,000$, a 6-minute walking test is added to measure the exercise capacity. ${ }^{2}$

\section{Step 7: translation into strategies to monitor implementation}

The diagnostic pathway was implemented for patients with asthma and COPD (Figure 2).

We developed two strategies to monitor implementation of the comprehensive assessment; (these data will be described elsewhere). In short: the first is an electronic clinical chart review to check which measurements were actually performed. The results of this chart review are systematically recorded in the newly developed Integral Practice Analysis Tool (IPAT). Implementation will also be monitored using quantitative data from the hospital administrative system. Data will be extracted with regard to adherence to the program by the team, the existing diagnostic variation between the pulmonologists, and the follow-up treatment in primary and secondary care.

\section{Step 8: implementation and monitoring in a multidisciplinary team}

The result, a 2-day stepped diagnostic pathway, includes a basic comprehensive health status assessment with a stepwise 


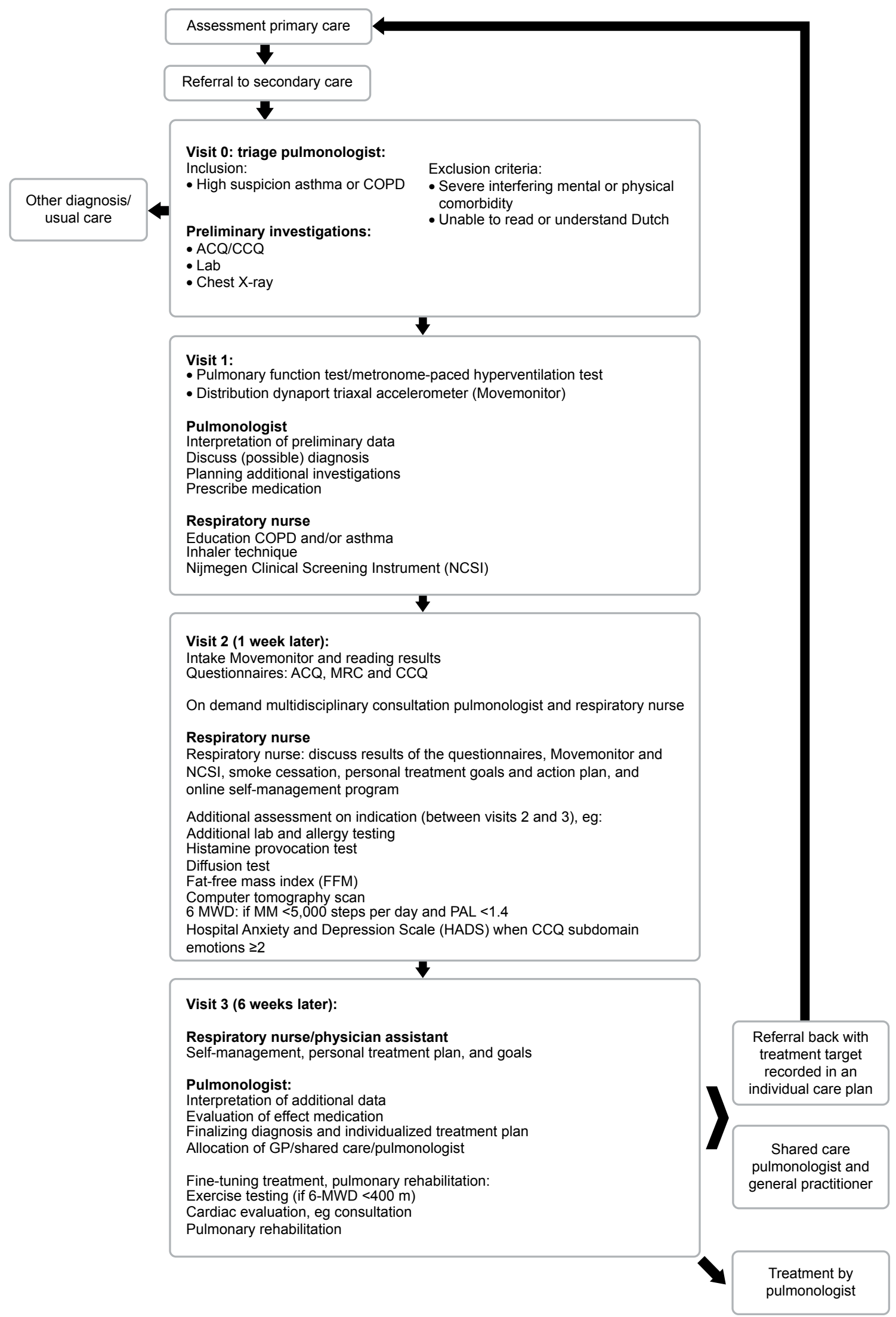

Figure 2 Flowchart of the diagnostic pathway.

Abbreviations: ACQ, Asthma Control Questionnaire; GP, general practitioner; MRC, Medical Research Council; CCQ, Clinical COPD Questionnaire; 6-MWD, 6-minute walk distance; MM, move monitor; PAL, physical activity level. 
implementation in the respiratory outpatient department of a large teaching hospital (Sint Franciscus Gasthuis, Rotterdam, the Netherlands). Prior to implementation, each discipline (separately) received training in specific areas. The implementation occurred stepwise to allow any necessary adjustments. The expert team discussed the logistics process on a monthly basis. In addition, every 6 weeks, the respiratory team evaluated the content of the assessment. All disciplines were requested to provide feedback on a regular basis; this resulted in some minor adjustments. The single most important change was the addition of an extra third visit after 6 weeks; this is because, at 1 week, the diagnostic trajectory was too short to start specific therapeutic procedures that include behavioral change.

\section{Discussion}

A diagnostic pathway was designed to provide a comprehensive overview of the patient's health status to identify areas of behavioral improvement, and set personal targets for therapy based on shared decision-making. This innovative assessment schedule was successfully implemented in the daily routine care of a local outpatient pulmonary department.

International experts increasingly acknowledge the need and usefulness of an integrated care concept to improve the care for patients with obstructive pulmonary disease. ${ }^{9}{ }^{10}$ However, international guidelines lack structured information on a diagnostic pathway for patients with more severe disease. ${ }^{1,12}$ In the absence of guidelines, we developed the content of the diagnostic process for newly referred patients suspected to have obstructive lung disease that could be implemented in routine care in an outpatient pulmonary department.

Although the content of the diagnostic package seems to be relatively extensive, individual consequences of obstructive airway diseases are complex, show significant variation between patients, and cannot be reliably predicted from biomedical measurements such as $\mathrm{FEV}_{1}$. This was confirmed by the results of the Delphi procedure, the opinions of the experts, and the evidence-based therapeutic implications.

Three novel diagnostics were incorporated in the pathway, ie, the MPH test to measure dynamic hyperinflation, an activity monitor to objectively evaluate physical activity in daily life, and the NCSI as a comprehensive assessment tool to gain detailed insight into symptoms, functional limitations, and quality of life.

Dynamic hyperinflation, but not airflow obstruction, is an increasingly important indicator of lung function impairment with clinical consequences for dyspnea, exercise capacity, and physical activity in COPD. Evidence-based therapeutic options are available to attenuate dynamic hyperinflation, including the use of a (long-acting) bronchodilator, exercise training, and breathing retraining. ${ }^{26}$

Physical inactivity is an extrapulmonary manifestation of obstructive lung disease and an important determinant of the burden of disease. A low physical activity should raise the question whether the patient is not willing to, or is not able to, exercise. The easiest way to assess physical activity is by use of a questionnaire. However, this may lead to significant over- or underestimation of physical activity. Reliable measures of physical activity can be obtained with activity monitors, like the Dynaport. This not only provides information on the number of steps/day, but also on the intensity of exercise, energy spent, and the time spent sitting or lying. All of these activities are known to be independent components of health and all can be a target for nonmedical intervention.

Successful adaptation to a chronic illness helps patients reduce their burden of disease and participate in social activities, despite existing physical limitations. Interventions, which correspond with this burden and focus on the patient needs, should support behavior change. ${ }^{34}$

In this respect, health-related quality of life questionnaires are essential to obtain comprehensive insight into the impact of the disease on a patient's life. Therefore, we introduced our third innovative diagnostic tool: the NCSI. This tool captures all four domains (physiological functioning, symptoms, functional impairment, and quality of life) by measuring multiple subdomains in a quick and detailed assessment. The results of this assessment are presented visually. It provides an indepth view of the implications of the disease for the patient and forms the starting point of a discussion on the patient's role and personal goals, with subsequent treatment options to reach these goals.

This shared decision-making process results in a written individual care plan to which patients and professionals feel committed and motivates the patient to start working on these goals.

Although we were successful in the development and implementation of an innovative diagnostic pathway for patients with obstructive lung disease, the added value still needs to be established.

The next step is to conduct a study on the effects of the introduction of the diagnostic pathway on the application of the principles of integrated care. In the longer term, if the diagnostic pathway is well implemented and collaboration with primary care is further established, the added value for outcomes relevant to patients needs to be proved by 
a clinical study. Therefore, a necessary prerequisite is to develop and implement an effective intervention program in which interventions are offered to patients in line with the analysis of health status.

\section{Conclusion}

We succeeded in developing and implementing an innovative diagnostic pathway for patients with obstructive lung disease referred to secondary care. As this diagnostic pathway aims to perform a comprehensive analysis of health status, it focuses not only on biomedical aspects of the disease but also reviews behavioral aspects that provide further information on the individual patient's health status. Additional studies are required to determine the added value of the diagnostic pathway from an organizational perspective and from the individual patient's viewpoint.

\section{Acknowledgments}

The authors wish to thank "PICASSO for COPD" and the "Marga Roubos legacy" for funding this study.

\section{Disclosure}

The authors report no conflicts of interest in this work.

\section{References}

1. Global Initiative for Chronic Obstructive Pulmonary Disease (GOLD). Global Strategy for Diagnosis, Management and Prevention of COPD; 2014. Available from: http://www.goldcopd.org/guidelines-globalstrategy-for-diagnosis-management.html. Accessed February 3, 2015.

2. Watz H, Pitta F, Rochester CL, et al. An official European Respiratory Society statement on physical activity in COPD. Eur Respir J. 2014; 44:1521-1537.

3. Vercoulen JH. A simple method to enable patient-tailored treatment and to motivate the patient to change behaviour. Chron Respir Dis. 2012;9:259-268.

4. Wechsler ME. Managing asthma in primary care: putting new guideline recommendations into context. Mayo Clin Proc. 2009;84:707-717.

5. Wilson IB, Cleary PD. Linking clinical variables with health-related quality of life. A conceptual model of patient outcomes. JAMA. 1995; 273:59-65.

6. Vercoulen JH, Daudey L, Molema J, et al. An integral assessment framework of health status in chronic obstructive pulmonary disease (COPD). Int J Behav Med. 2008;15:263-279.

7. Spruit MA, Singh SJ, Garvey C, et al. An official American Thoracic Society/European Respiratory Society statement: key concepts and advances in pulmonary rehabilitation. Am J Respir Crit Care Med. 2013; 188(8):e13-e64.

8. Long Alliantie Nederland. Zorgstandaard COPD [Care Standard COPD]. Amersfoort, The Netherlands: Long Alliantie Nederland; 2010. Dutch.

9. Nici L, ZuWallack R; American Thoracic Society Subcommittee on Integrated Care of the CP. An official American Thoracic Society workshop report: the Integrated Care of The COPD Patient. Proc Am Thorac Soc. 2012;9:9-18.

10. Agusti A, MacNee W. The COPD control panel: towards personalised medicine in COPD. Thorax. 2013;68:687-690.
11. Reuben DB, Tinetti ME. Goal-oriented patient care - an alternative health outcomes paradigm. $N$ Engl J Med. 2012;366:777-779.

12. Global Initiative for Asthma. Global strategy for asthma management and prevention; 2011. Available from: http://www.ginasthma.org/ local/uploads/files/GINA_Report2011_May4.pdf. Accessed October 9, 2015.

13. Effing TW, Bourbeau J, Vercoulen J, et al. Self-management programmes for COPD: moving forward. Chron Respir Dis. 2012;9(1):27-35.

14. Lodewijckx C, Decramer M, Sermeus W, Panella M, Deneckere S, Vanhaecht K. Eight-step method to build the clinical content of an evidence-based care pathway: the case for COPD exacerbation. Trials. 2012;13:229.

15. Tsiachristas A, Cramm JM, Nieboer AP, Rutten-van Molken MP. Changes in costs and effects after the implementation of disease management programs in the Netherlands: variability and determinants. Cost Eff Resour Alloc. 2014;12:17.

16. Long Alliantie Nederland. Zorgstandaard Astma [Care Standard Asthma]. Amersfoort, The Netherlands: Long Alliantie Nederland; 2012. Dutch.

17. Jones P, Miravitlles M, van der Molsen T, Kulich K. Beyond FEV(1) in COPD: a review of patient-reported outcomes and their measurement. Int J Chron Obstruct Pulmon Dis. 2012;7:697-709.

18. Glaab T, Vogelmeier C, Buhl R. Outcome measures in chronic obstructive pulmonary disease (COPD): strengths and limitations. Respir Res. 2010;11:79.

19. Spruit MA. Pulmonary rehabilitation. Eur Respir Rev. 2014;23:55-63.

20. Fitch K, Bernstein SJ, Aguilar MD, et al. The RAND/UCLA appropriateness method user's manual. Santa Monica, CA: RAND. Available from: http://www.rand.org/publications/MR/MR1269/. Accessed October 10, 2015.

21. Vanfleteren LE, Spruit MA, Groenen M, et al. Clusters of comorbidities based on validated objective measurements and systemic inflammation in patients with chronic obstructive pulmonary disease. Am J Respir Crit Care Med. 2013;187:728-735.

22. Prescott E, Almdal T, Mikkelsen KL, Tofteng CL, Vestbo J, Lange P. Prognostic value of weight change in chronic obstructive pulmonary disease: results from the Copenhagen City Heart Study. Eur Respir J. 2002;20:539-544.

23. Lv N, Xiao L, Camargo CA Jr, et al. Abdominal and general adiposity and level of asthma control in adults with uncontrolled asthma. Ann Am Thorac Soc. 2014;11:1218-1224.

24. Rang LC, Murray HE, Wells GA, Macgougan CK. Can peripheral venous blood gases replace arterial blood gases in emergency department patients? CJEM. 2002;4:7-15.

25. van Dijk WD, van Bemt L, van den Haak-Rongen S, et al. Multidimensional prognostic indices for use in COPD patient care. Respir Res. 2011;12:151.

26. Gelb AF, Gutierrez CA, Weisman IM, Newsom R, Taylor CF, Zamel N. Simplified detection of dynamic hyperinflation. Chest. 2004; 126:1855-1860.

27. Dweik RA, Boggs PB, Erzurum SC, et al. An official ATS clinical practice guideline: interpretation of exhaled nitric oxide levels (FENO) for clinical applications. Am J Respir Crit Care Med. 2011; 184:602-615.

28. Bestall JC, Paul EA, Garrod R, Garnham R, Jones PW, Wedzicha JA. Usefulness of the Medical Research Council (MRC) dyspnoea scale as a measure of disability in patients with chronic obstructive pulmonary disease. Thorax. 1999;54:581-586.

29. van Dam van Isselt EF, Spruit M, Groenewegen-Sipkema KH, Chavannes NH, Achterberg WP. Health status measured by the Clinical COPD Questionnaire (CCQ) improves following post-acute pulmonary rehabilitation in patients with advanced COPD: a prospective observational study. NPJ Prim Care Respir Med. 2014;24:14007.

30. Peters JB, Rijssenbeek-Nouwens LH, Bron AO, et al. Health status measurement in patients with severe asthma. Respir Med. 2014;108: $278-286$. 
31. Van Remoortel H, Raste Y, Louvaris Z, et al. Validity of six activity monitors in chronic obstructive pulmonary disease: a comparison with indirect calorimetry. PLoS One. 2012;7:e39198.

32. Sundh J, Stallberg B, Lisspers K, Montgomery SM, Janson C. Comorbidity, body mass index and quality of life in COPD using the Clinical COPD Questionnaire. COPD. 2011;8:173-181.
33. Juniper EF, Guyatt GH, Ferrie PJ, Griffith LE. Measuring quality of life in asthma. Am Rev Respir Dis. 1993;147:832-838.

34. Huber M, Knottnerus JA, Green L, et al. How should we define health? BMJ. 2011;343:d4163. 


\section{Supplementary material}

Table SI Delphi consensus aspects

\begin{tabular}{|c|c|c|}
\hline No & Determinants & Percentage \\
\hline $\mathrm{I}$ & Lung function & 98.7 \\
\hline 2 & Comorbidities & 97.5 \\
\hline 3 & Pulmonary medication & 97.5 \\
\hline 4 & Respiratory complaints & 97.5 \\
\hline 5 & Pulmonary history & 96.3 \\
\hline 6 & Hospital admission last year as a result of exacerbations & 96.3 \\
\hline 7 & ICU admission last year as a result of exacerbations & 95.0 \\
\hline 8 & Physical activity, subjective & 95.0 \\
\hline 9 & Exacerbation frequency last year & 93.8 \\
\hline 10 & Airway obstruction and reversibility & 93.8 \\
\hline 11 & Smoking status & 92.5 \\
\hline 12 & Physical examination & 91.5 \\
\hline 13 & Physical activity, objective & 90.0 \\
\hline 14 & Nonpulmonary medication & 88.8 \\
\hline 15 & Nutritional status & 88.8 \\
\hline 16 & Allergic symptoms & 88.8 \\
\hline 17 & Quality of life, objective & 88.8 \\
\hline 18 & Allergy research (asthma) & 87.5 \\
\hline 19 & Allergy, subjective & 86.3 \\
\hline 20 & Lung volume (restriction, static hyperinflation) & 86.3 \\
\hline 21 & Cardiac complaints & 86.3 \\
\hline 22 & Exposition & 82.5 \\
\hline 23 & Classification of COPD in GOLD & 81.3 \\
\hline 24 & Diffusion capacity & 81.3 \\
\hline 25 & Anxiety and depression & 81.3 \\
\hline 26 & Exercise capacity & 80.0 \\
\hline 27 & Quality of life, subjective & 80.0 \\
\hline 28 & Dynamic hyperinflation & 78.8 \\
\hline 29 & Thorax X-ray & 76.3 \\
\hline
\end{tabular}

Notes: Of the $4 \mathrm{I}$ selected components for the diagnostic assessment, consensus was reached for 29 components. Consensus was reached when $\geq 75 \%$ of the respondents selected "(very) important" for a specific component rated on a seven-point Likert scale.

Abbreviations: ICU, intensive care unit; COPD, chronic obstructive pulmonary disease; GOLD, global initiative for chronic obstructive pulmonary disease.

\section{Publish your work in this journal}

The International Journal of COPD is an international, peer-reviewed journal of therapeutics and pharmacology focusing on concise rapid reporting of clinical studies and reviews in COPD. Special focus is given to the pathophysiological processes underlying the disease, intervention programs, patient focused education, and self management protocols.

\section{Dovepress}

This journal is indexed on PubMed Central, MedLine and CAS. The manuscript management system is completely online and includes a very quick and fair peer-review system, which is all easy to use. Visit $\mathrm{http} / / / \mathrm{www}$.dovepress.com/testimonials.php to read real quotes from published authors. 